\title{
Efficacy of triamcinolone injection with or without oral meloxicam for treatment of anserine syndrome: a randomized, double-blind, placebo- controlled trial
}

\author{
S. Thiengwittayaporn ${ }^{1}$, S. Phatwong ${ }^{1}$, N. Kangkano ${ }^{2}$, N. Charoenphandhu' ${ }^{2,3,4}$ \\ 1 Department of Orthopaedics, Faculty of Medicine Vajira Hospital, Navamindradhiraj University, Dusit, Bangkok, \\ Thailand \\ 2 Center of Calcium and Bone Research (COCAB), and the Department of Physiology, Faculty of Science, \\ Mahidol University, Bangkok, Thailand \\ 3 Institute of Molecular Biosciences, Mahidol University, Nakhon Pathom, Thailand \\ 4 The Academy of Science, The Royal Society of Thailand, Dusit, Bangkok, Thailand
}

\section{CORRESPONDING AUTHOR:}

Satit Thiengwittayaporn

Department of Orthopaedics

Faculty of Medicine Vajira Hospital

Navamindradhiraj University

681 Samsen Rd, Dusit,

Bangkok 10300, Thailand

Phone: +66 22443376

Fax: +6622436106

DOI:

10.32098/mltj.01.2019.21

\begin{abstract}
SUMMARY
Objectives. To compare the effectiveness and safety of oral meloxicam after triamcinolone acetonide injection alone for treatment of the anserine syndrome. Methods. A randomized, double-blind placebo-controlled trial was conducted in 64 patients with anserine syndrome given an injection of $20 \mathrm{mg}$ of triamcinolone acetonide. The patients were randomly separated into two groups: Group A received oral meloxicam for 7 days $(n=32)$ and Group B received placebo tablets for 7 days $(n=32)$. At three weeks after the injection, primary outcomes (patient's symptoms, physical signs and pain visual analog scale) and adverse reactions were assessed by an independent, blinded evaluator. Results. The success rates were 50\% and $40.6 \%$ for Groups $A$ and B, respectively. No significant difference of the success rates between the two groups was observed $(p=0.62)$. Common adverse reactions were found to be pain after the injection and dyspepsia. Conclusions. Injection of triamcinolone acetonide seems to be sufficient and safe to treat the anserine syndrome. The adding of oral meloxicam, and perhaps other NSAIDs, does not improve the efficacy of triamcinolone acetonide in the treatment of the anserine syndrome.
\end{abstract}

\section{KEY WORDS}

anserine syndrome; steroid injection; non-steroidal anti-inflammatory drugs; NSAIDs ; anserine bursitis

\section{INTRODUCTION}

A common complaint in clinical practice of anserine syndrome is pain in the region of pes anserinus insertion, consisting of sartorius, gracilis, and semitendinosus tendons, approximately 5 centimeters distally of the medial part of the knee $(1,2)$. Up to now, the anatomical validity of this disease is still controversial whether it is as an inflammatory condition of the bursa and/or tendon, thus this condition is termed "anserine syndrome" (1-7) Classic characteristics symptoms are vague medial knee pain or tenderness and swelling along the proximal medial tibia, mimicking medial meniscus/medial collateral ligament injury (8). The symptoms may be exacerbated when the patient ascends or descends the stairs. So far, there has not been any study on the real prevalence and risk factors. However, some reports suggest that this syndrome is more common in overweight females with osteoarthritis of the knees $(1,3,9)$. Although the previous observations showed the prevalence of anserine bursitis in non-insulin dependent diabetic patients is common (10), another study used ultrasound (US) to investigate the knee pain in diabetic patients with anserine bursitis, and found structural changes such as meniscal lesions 
from osteoarthritis, which might be the cause of their pain (11). Other conditions associated with pes anserine bursitis include degenerative joint disease of the knee, obesity, valgus knee deformity, pes planus, and sporting activities $(2,12,13)$. Typically, the diagnosis of this syndrome is based on the symptoms, which include pain in the medial aspect of the knee when going upstairs or downstairs, sensitivity to palpation (digital pressure) on the area of insertion, and, occasionally, local edema $(8,14,15)$, but diagnostic studies such as US, computed tomography (CT) and magnetic resonance imaging (MRI) can be used to evaluate this condition if the diagnosis is in question $(5,11,16-19)$. In contrast, some literatures reported that even with normal diagnosis results from these diagnostic studies, the symptoms and physical examination findings consistent with this syndrome were found $(5,6,11,17,20,21)$.

There is no definite consensus for the treatments of this syndrome; however, the common treatments in clinical practice include physiotherapy, exercise therapy, especially eccentric exercise $(22,23)$, oral administration of non-steroidal anti-inflammatory drugs (NSAIDs) alone, and steroid injection with or without NSAIDs (24). A previous study has found a significant improvement of anserine bursitis in corticosteroid injection group when compared to NSAIDs group (14). Another study by Larsson and Baum (25) reported that approximately $70 \%$ of corticosteroid injected knees showed significant improvement. However, there is improbability that oral administration of NSAIDs after steroid injection improves the success rates of the treatment for the anserine syndrome.

We conducted a randomized, double-blind, placebo-controlled trial to examine effectiveness and safety of oral meloxicam after triamcinolone acetonide injection, compared with triamcinolone acetonide injection alone for treatment of the syndrome. We hypothesized that there would be no significant difference in the success rates when anserine syndrome was treated with triamcinolone acetonide injection, with or without oral meloxicam.

\section{MATERIALS AND METHODS}

This prospective, randomized, controlled study was approved by the ethical committee of our institution (COA 24/2557; approved date 30/04/14). All authors conducted this study ethically according to international standards (26). Sixty-eight patients with the clinical diagnosis of anserine syndrome were enrolled into the study. The diagnosis of anserine syndrome was established when the patients met the two criteria of symptom and physical sign: 1) symptom: pain in the medial aspect of the knee when going upstairs or downstairs; 2) physical sign: tender when applying digital pressure on the area of insertion (medial aspect of the knee) (14). We excluded patients who had intraarticular pathology reflecting the medial pain in the knee, such as meniscal disease, medial collateral ligament injury, medial plicature, previous treatment with steroid injection (injection for anserine syndrome and also for intra-articular knee injection), previous treatment with peri-articular and intra-articular platelet-rich plasma (PRP) or hyaluronic acid injection or laser therapy, were pregnant or lactating, and had contraindication to the use of NSAIDs, steroid injection and lidocaine injection. We also excluded the patients if they had previously taken NSAIDs or received an oral steroid injection in the past 2 weeks. We did not exclude the patients with concomitant knee osteoarthritis and diabetes mellitus. All included patients with anserine syndrome were given an injection of $2 \mathrm{~mL}(20 \mathrm{mg}$ ) of triamcinolone acetonide (Shincort $^{\circledR} 10 \mathrm{mg} / \mathrm{mL}$, Yung Shin Pharmaceutical Industrial, Taiwan) and $2 \mathrm{~mL}$ of $1 \%$ lidocaine without adrenaline (Astra Pharma, Mississauga, Ontario, Canada). The injection technique $(17,27)$ was performed in the most painful spot at the medial aspect of the knee using an aseptic technique by a certified orthopedist who was not the evaluator (figure 1). Patients were randomly assigned to each of the two arms with random permuted blocks of four. The patients were randomly separated into two groups: Group A received oral

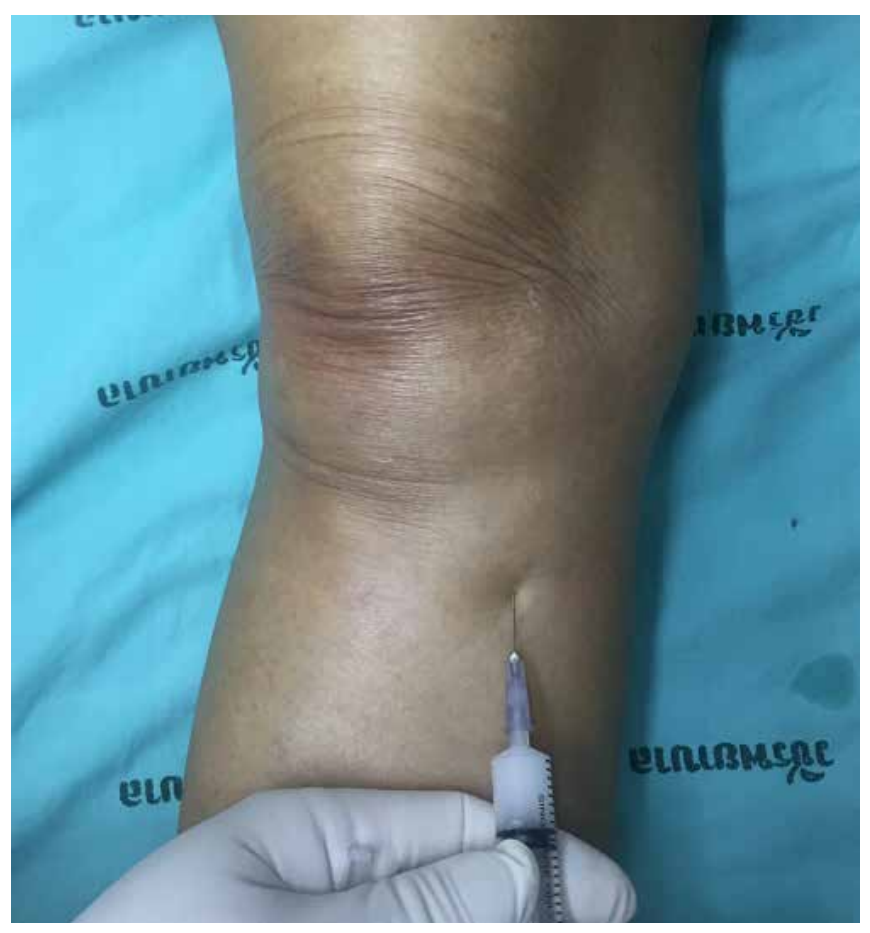

Figure 1 - The triamcinolone acetonide injection was performed in the most painful spot at the medial aspect of the knee. 
meloxicam (Mobic ${ }^{\circledR} .5 \mathrm{mg}$, Boehringer Ingelheim, Indonesia, under license of Boehringer Ingelheim, Germany) once daily for 7 days; Group B received placebo tablets once daily for 7 days. The patients flow is summarized in figure 2 . Both the patients and the investigators measuring the final outcomes were blinded with regard to the trial arm to which the patient had been assigned. The patients were encouraged to avoid going upstairs or downstairs. All patients were discouraged to receive other NSAIDs, analgesic drugs, and steroid injections. The patients who had pes planus or genus valgus were encouraged to use the plantar orthoses.

Demographic data including age, sex, body mass index, number of co-morbidities, side, duration of symptoms before treatment, previous treatment, and pain visual analogue scale (VAS) were recorded at the time of first visit. The initial follow-up was performed one week after the injection to record and treat for any adverse reactions. At three weeks after the injection, primary outcomes (patient's symptoms, physical signs and pain VAS) and adverse events were assessed by an independent, blinded evaluator. Subsequent follow-ups were performed every 3 months, or when patient's symptom recurred to evaluate the recurrence rate. The primary outcome measures compose of (1) patient's symptoms: pain in the medial aspect of the knee when going upstairs or downstairs, (2) physical signs: tender when apply digital pressure on the area of insertion (medial aspect of the knee) and (3) pain VAS.

Pain at initial visit was evaluated by a $100 \mathrm{~mm}$ long visual analog scale, with 0 indicating no pain and 100 indicating extreme pain. At three weeks after the injection, the patients were asked to compare the pain with the pain before treatment. The evaluation was performed with a $200 \mathrm{~mm}$ long visual analog scale, with 0 at the center indicating no change in pain. Value to the right indicated improvement or decrease in pain, with an end at 100 indicating no pain. Value to the left indicated deterioration or increase in pain, with an end at -100 indicating worst pain. The patient's outcome was graded as a success if patient's symptoms and physical signs were completely resolved, and the improvement in the pain VAS was $>70 \%$. The patient's outcome was graded as a failure if patient's symptoms or physical signs were not completely resolved, or improvement in the pain VAS was $<70 \%$. The patient's outcome was graded as an improvement if one of any categories of patient's symptoms or physical signs was completely resolved or improvement in the pain VAS.

\section{Statistical analysis}

The sample size was estimated to be 26 patients per group to ensure sufficient power of 80 with a significant difference ( $\alpha=0.05$, two-sided significance level). In order to

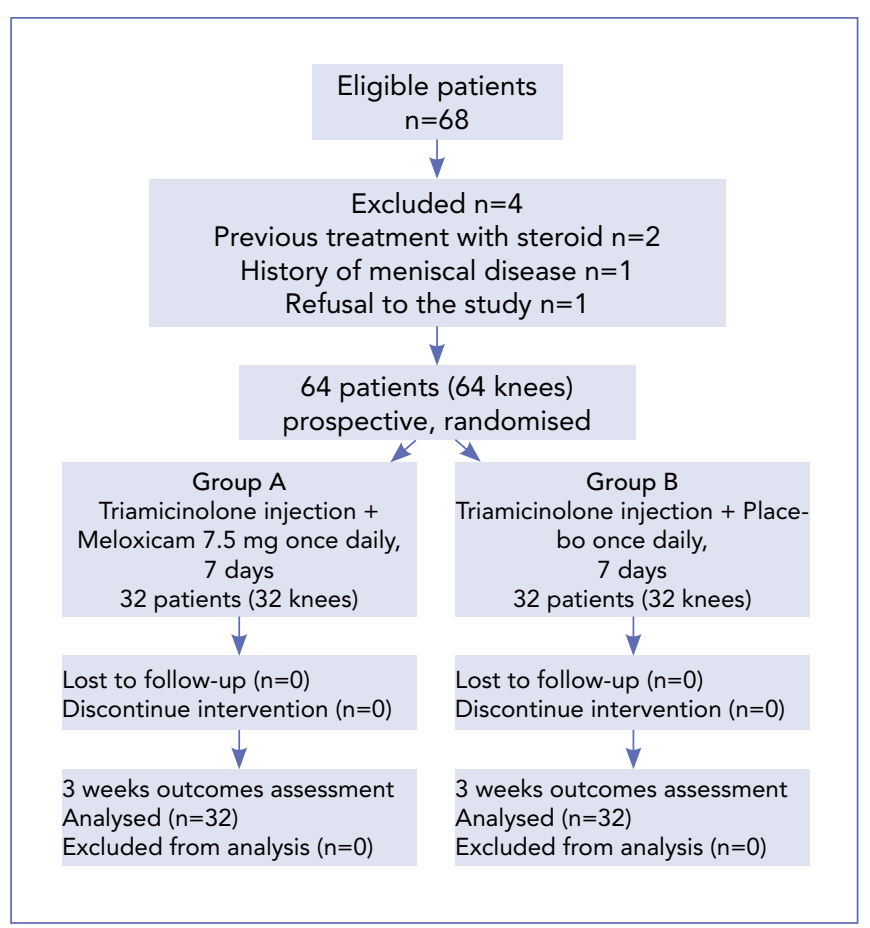

Figure 2 - The study CONSORT chart.

compensate for $10 \%$ of patients who might not follow through a follow-up appointment, we recruited at least 29 knees per group to ensure sufficient sample size to reach the significant level.

Demographic data of both groups were compared with an independent $\mathrm{t}$-test (student $\mathrm{t}$-test) and chi-square. The clinical outcomes of both groups were compared with an independent t-test and chi-square. End point data were analyzed according to the intention-to-treat principle. The p-value $<0.05$ indicates a statistically significant difference (SPSS version 21, SPSS Inc, Chicago, IL, USA).

\section{RESULTS}

From May 2014 to July 2016, a total of 68 patients were diagnosed with anserine syndrome. Four patients were excluded from the study because of their previous treatment with steroid in 2 , history of meniscal disease in 1 , and refusal to the study in 1 . The group treated with triamcinolone acetonide injection and oral meloxicam (Group A) consisted of 28 female and 4 male who had an average age of 54.6 years (a range of 35 - 71 years). The group treated with triamcinolone acetonide injection and oral placebo (Group B) consisted of 25 female and 7 male who had an average age of 54.7 years (a range of 36-72 years). Others demographic variables such as gender, body mass index 
(BMI), duration of symptom, and pain score on visual analogue scale before treatment were similar between the two groups (table I).

\section{Primary outcomes}

The success rates (completely resolved patient's symptoms, completely resolved physical signs, and $>70 \%$ improvement in the pain VAS) assessed at three weeks after the injection showed no significant difference between the two groups. The patient's symptoms assessed by pain in the medial aspect of the knee when going upstairs or downstairs were not significantly different between the two groups. The physical signs, assessed by tender when apply digital pressure on the area of insertion at approximately 5 centimeters distally of the medial part of the knee, were not significantly different between the two groups. The pain improvement score on visual analogue scale after treatment was also not significantly different between the two groups. However, there were improvements of the syndrome after treatment in both groups $90.6 \%$ and $80.4 \%$ for group A and group B, respectively) but the improvements were not significantly different between the two groups. The overall primary outcomes are shown in table II.

\section{Secondary outcomes}

The recurrence rate evaluated in the patients whose successful treatment were not significantly different between the two groups. The averaged times to recurrence for Group A and Group B were 16.0 (4 to 24 ) weeks and 18.7 (4 to 28) weeks, respectively. These times were not significantly different between the two groups. The prevalence of adverse events was not significantly different between the two groups. There were no serious adverse events found in this study. The most common adverse reaction to triamcinolone acetonide injection was pain at the infiltration site. The most common adverse reaction to meloxicam was dyspepsia. The overall secondary outcomes and adverse events are shown in table III.

\section{DISCUSSION}

Previously, treatment of anserine syndrome with steroid injection have shown significant improvement in approximately $70 \%$ of injected knees (25). Another study examined 44 consecutive patients with anserine bursitis treated with $500 \mathrm{mg}$ of naproxen every 12 hours compared with corticosteroid injection and used verbal pain scale to evaluate the effectiveness of the treatment regimes. It found a

Table I - Demographic data.

\begin{tabular}{|c|c|c|c|}
\hline & Group A $(n=32)$ & Group B $(n=32)$ & p value \\
\hline age (year) & $54.6(35$ to 71$)$ & $54.7(36$ to 72$)$ & 0.96 \\
\hline body mass index & $29.4(21.9$ to 39.0$)$ & $30.2(22.0$ to 40.1$)$ & 0.53 \\
\hline number of co-morbidities & $1.6(0$ to 4$)$ & $1.2(0$ to 4$)$ & 0.19 \\
\hline left : right & $18: 14$ & $15: 17$ & 0.62 \\
\hline
\end{tabular}

Table II - Primary outcomes.

\begin{tabular}{|c|c|c|c|}
\hline & Group A $(n=32)$ & Group B $(n=32)$ & p value \\
\hline $\begin{array}{l}\text { patient's symptoms: pain in the medial aspect of the knee } \\
\text { when going upstairs or downstairs (pain : no pain) }\end{array}$ & $13: 19$ & $8: 24$ & 0.29 \\
\hline $\begin{array}{l}\text { pain improvement score on visual analogue scale after } \\
\text { treatment (points) }\end{array}$ & $72.9(0$ to 100$)$ & 74.1 (20 to 100$)$ & 0.78 \\
\hline Improvement (no.) (\%) & $29(90.6)$ & $27(84.4)$ & 0.71 \\
\hline
\end{tabular}


Table III - Secondary outcomes.

\begin{tabular}{llll}
\hline & Group A (n= 32) & Group B (n= 32) & p value \\
\hline recurrence no. (\%) & $4(25)$ & $3(23)$ & 1.00 \\
\hline time to recurrence (week) & $16.0(4$ to 24) & $18.7(4$ to 28) & 0.62 \\
\hline Adverse event & & & 1.00 \\
\hline triamcinolone acetonide & 13 & 14 & \\
- pain & 9 & 10 & \\
- ecchymosis & 3 & 4 & 0.10 \\
- hypopigmentation & 1 & 0 & \\
\hline meloxicam & 6 & 1 & \\
- dyspepsia & 4 & 0 & \\
- edema & 2 & & \\
\hline
\end{tabular}

significant improvement in $58 \%$ and resolution in $5 \%$ in the naproxen group, compared with $70 \%$ of significant improvement and $30 \%$ resolution in the corticosteroid group (14). In contrast, one clinical trial randomized 58 patients with anserine syndrome to receive an infiltration of lidocaine plus $40 \mathrm{mg}$ methylprednisolone or lidocaine plus distilled water, and evaluated the outcomes using the WOMAC scale. Both groups received $100 \mathrm{mg}$ diclofenac sodium for 10 days. There was no statistical difference of WOMAC score between both groups with an improvement of $61.6 \%$ and $62.8 \%$, respectively. They concluded that the use of an infiltration of methylprednisolone fails to achieve improvement at 4 weeks in patients with anserine syndrome taking diclofenac compared to placebo (28). To the best of our knowledge, there is no systemic study evaluating effectiveness of the addition of NSAIDs with steroid injection to treatment of anserine syndrome.

Regarding to the success rate and improvement outcomes in this study, the group of triamcinolone acetonide injection and oral meloxicam seemed to have superior outcomes to the group of triamcinolone acetonide injection and placebo $(50 \%$ and $40.6 \%$, respectively) but no significant difference. Improvement of the syndrome was found in $90.6 \%$ for triamcinolone acetonide injection adding oral meloxicam and $84.4 \%$ for triamcinolone acetonide injection alone, without statistical difference between the groups.

For our secondary outcomes, the recurrence rates of the patients whose treatment was successful were $25 \%$ and $23 \%$, with averaged times to recurrence of 16.0 weeks and 18.7 weeks for triamcinolone acetonide injection plus meloxicam group and triamcinolone acetonide injection plus placebo group, respectively. There was also no significant difference between the groups. To our knowledge, this is the first clinical trial that reports the recurrence rate and averaged time to recurrence.
When compared with other previous studies, our success rate was lower. This was probably because our criteria of successful outcomes were strict to the patient's symptoms, physical signs and percentage of improvement in the pain VAS, with success graded by complete resolve of patient's symptoms and physical signs, and $>70 \%$ improvement in the pain VAS. In addition, other studies defined the improvement outcomes by WOMAC score or by verbal pain score only.

In the present study, the most common adverse event of triamcinolone acetonide injection was pain at the infiltration site, whereas the most common adverse event of oral meloxicam was dyspepsia. There was no serious adverse event from triamcinolone acetonide injection and oral meloxicam found in this study. We chose the meloxicam as a representative of the other NSAIDs because of its low gastrointestinal side effect and long acting effect, which allowed a oncea-day dosage only (29).

There are several limitations to this study. First, this study used only patient's symptoms, physical signs and pain visual analog scale as the diagnosis criteria. We did not perform an US evaluation to confirm the diagnosis of anserine syndrome because of our limitation in instruments and specialists, whereas we used patient's symptoms, physical signs and pain visual analog scale as strict criteria to diagnose. Second, this study did not use the Western Ontario and McMaster Universities Arthritis Index (WOMAC) or the Knee injury and Osteoarthritis Outcome Score (KOOS) to evaluate the outcome measure, whereas we used $>70 \%$ improvement in the pain VAS as criteria to focus on pain level. Third, with the limitation in the US instrument and the specialists, this study used a landmark-based injection technique to inject the triamcinolone acetonide instead of an ultrasound-guided injection technique even though it was reportedly less accurate (16). However, we made the best attempt to inject 
at the most tender point which is associated with the landmark of anserine syndrome.

In conclusion, triamcinolone acetonide injection is sufficient and safe to treat anserine syndrome. The adding of oral meloxicam, and perhaps other NSAIDs, does not improve the efficacy of triamcinolone acetonide in the treatment of the anserine syndrome.

\section{Acknowledgments}

NC is the Thailand Research Fund (TRF) Senior Research Scholar (RTA6080007) and the investigator of TRF International Research Network Program (IRN60W0001) awarded by TRF. NK has received scholarship awarded from Thailand Graduate Institute of Science and Technology (TG-CPMO 01-56-008).

\section{Conflict of Interest}

On behalf of all authors, the corresponding author states that there is no conflict of interest.

\section{Ethical approval}

All procedures performed in studies involving human participants were in accordance with the ethical standards of the institutional and/or national research committee and with the 1964 Helsinki declaration and its later amendments or comparable ethical standards.

\section{Informed consent}

Informed consent was obtained from all individual participants included in the study.

\section{REFERENCES}

1. Alvarez-Nemegyei J, Canoso JJ. Evidence-Based Soft Tissue Rheumatology IV: Anserine Bursitis. Journal of clinical rheumatology: practical reports on rheumatic and musculoskeletal diseases. 2004; 10(4):205-206.

2. Wood LR, Peat G, Thomas E, Duncan R. The contribution of selected non-articular conditions to knee pain severity and associated disability in older adults. Osteoarthritis and cartilage / OARS, Osteoarthritis Research Society 2008; 16(6):647-653.

3. Gnanadesigan N, Smith RL. Knee pain: osteoarthritis or anserine bursitis? J Am Med Dir Assoc 2003;4(3):164-166.

4. Rennie WJ, Saifuddin A. Pes anserine bursitis: incidence in symptomatic knees and clinical presentation. Skeletal Radiol 2005; 34(7):395-398.

5. Uson J, Aguado P, Bernad M, Mayordomo L, Naredo E, Balsa A, et al. Pes anserinus tendino-bursitis: what are we talking about? Scand J Rheumatol 2000; 29(3):184-186.
6. Kang I, Han SW. Anserine bursitis in patients with osteoarthritis of the knee. Southern Med J 2000; 93(2):207-209.

7. Huang TW, Wang CJ, Huang SC. Polyethylene-induced pes anserinus bursitis mimicking an infected total knee arthroplasty: a case report and review of the literature. J Arthroplasty 2003; 18(3):383-386.

8. Forbes JR, Helms CA, Janzen DL. Acute pes anserine bursitis: MR imaging. Radiology 1995; 194(2):525-527.

9. Handy JR. Anserine bursitis: a brief review. Southern Med J 1997; 90(4):376-377.

10. Cohen SE, Mahul O, Meir R, Rubinow A. Anserine bursitis and non-insulin dependent diabetes mellitus. Journal Rheumatol 1997; 24(11):2162-2165.

11. Unlu Z, Ozmen B, Tarhan S, Boyvoda S, Goktan C. Ultrasonographic evaluation of pes anserinus tendino-bursitis in patients with type 2 diabetes mellitus. Journal Rheumatol 2003; 30(2):352-354.

12. Uysal F, Akbal A, Gokmen F, Adam G, Resorlu M. Prevalence of pes anserine bursitis in symptomatic osteoarthritis patients: an ultrasonographic prospective study. Clin Rheumatol 2015; 34(3):529-533.

13. Alvarez-Nemegyei J. Risk factors for pes anserinus tendinitis / bursitis syndrome: a case control study. JCR: practical reports on rheumatic and musculoskeletal diseases. 2007; 13(2):63-65.

14. Helfenstein M, Jr., Kuromoto J. Anserine syndrome. Rev Bras Reumatol 2010; 50(3):313-327.

15. Klippel JH, Weyand CM, Crofford LJ, Stone JH, Arthritis Foundation. Primer on the rheumatic diseases. 12th ed. Atlanta, Ga.: Arthritis Foundation; 2001. xiii, 700 pp.

16. Finnoff JT, Nutz DJ, Henning PT, Hollman JH, Smith J. Accuracy of ultrasound-guided versus unguided pes anserinus bursa injections. PM\&R: the journal of injury, function, and rehabilitation 2010; 2(8):732-739.

17. Yoon HS, Kim SE, Suh YR, Seo YI, Kim HA. Correlation between ultrasonographic findings and the response to corticosteroid injection in pes anserinus tendinobursitis syndrome in knee osteoarthritis patients. J Korean Med Sci 2005; 20(1):109-112.

18. Marra MD, Crema MD, Chung M, Roemer FW, Hunter DJ, Zaim S, et al. MRI features of cystic lesions around the knee. Knee 2008; 15(6):423-438.

19. Voorneveld C, Arenson AM, Fam AG. Anserine bursal distention: diagnosis by ultrasonography and computed tomography. Arthritis Rheum 1989; 32(10):1335-1338.

20. de Miguel Mendieta E, Cobo Ibanez T, Uson Jaeger J, Bonilla Hernan G, Martin Mola E. Clinical and ultrasonographic findings related to knee pain in osteoarthritis. Osteoarthritis and cartilage / OARS, Osteoarthritis Research Society 2006; 14(6):540-544.

21. Hill CL, Gale DR, Chaisson CE, Skinner K, Kazis L, Gale ME, et al. Periarticular lesions detected on magnetic resonance imaging: prevalence in knees with and without symptoms. Arthritis Rheum 2003; 48(10):2836-2844.

22. Frizziero A, Vittadini F, Fusco A, Giombini A, Masiero S. Efficacy of eccentric exercise in lower limb tendinopathies in athletes. The J Sport Med Phys Fit 2016; 56(11):1352-1358.

23. Frizziero A, Trainito S, Oliva F, Nicoli Aldini N, Masiero S, Maffulli N. The role of eccentric exercise in sport injuries rehabilitation. Brit Med Bull 2014; 110(1):47-75. 
24. Arromdee E, Matteson EL. Bursitis: Common condition, uncommon challenge. J Musculoskelet Med 2001; 18:213-224.

25. Larsson LG, Baum J. The syndrome of anserina bursitis: an overlooked diagnosis. Arthritis Rheum 1985; 28(9):10621065.

26. Padulo J, Oliva F, Frizziero A, Maffulli N. Muscles, Ligaments and Tendons Journal - Basic principles and recommendations in clinical and field Science Research: 2016 Update. MLTJ 2016; 6(1):1-5.
27. Courtney P, Doherty M. Joint aspiration and injection and synovial fluid analysis. Best Pract Res Cl Rh 2013; 27(2):137-169.

28. Vega-Morales D, Esquivel-Valerio JA, Negrete-Lopez R, Galarza-Delgado DA, Garza-Elizondo MA. Safety and efficacy of methylprednisolone infiltration in anserine syndrome treatment. Reumatologia Clinica 2012; 8(2):63-67.

29. Distel M, Mueller C, Bluhmki E, Fries J. Safety of meloxicam: a global analysis of clinical trials. Brit J Rheumatol 1996; 35Suppl1:68-77. 